\title{
Domogis: prototipo de un interfaz del sistema de control de un edificio integrado en un SIG
}

\author{
Domogis: prototype of a interface of a building control \\ system integrated into a GIS \\ M. Álvarez $z^{(*)}$ Á. Arquero ${ }^{(*)}$, E. Martínez ${ }^{(*)}$, O. Río ${ }^{(*)}$
}

RESUMEN

SUMMARY

Este trabajo trata de la utilización de los Sistemas de Información Geográfica (SIG) en uno de las nuevos requerimientos de la arquitectura, el control domótico. El objetivo es el desarrollo de un interfaz de comunicación del Sistema de Control de un Edificio (SCE) integrado en un SIG. La consecución de este objetivo implica previamente el desarrollo del SIG del Campus de Montegancedo sede de la Facultad de Informática de la UPM y la creación de un interfaz integrado en el SIG, desarrollado en lenguaje de programacion C\# de Microsoft. Este interfaz dirige al usuario en la realización de ciertas tareas de control domótico de las instalaciones urbanas y edificios del Campus universitario, como evaluar, monotorizar y gestionar datos procedentes de sensores estratégicamente situados en dicho Campus.

\section{6-18}

Palabras clave: Sistemas de Información Geografíca (SIG), Domotica, Control de edificios, Sensores.

\section{INTRODUCCIÓN}

Una de las primeras necesidades del hombre en su lucha por resguardarse y asegurar la subsistencia fue la de construir refugios donde descansar, asi como explorar y reconocer el territorio; saber dónde estaba, adónde tenía que desplazarse y cómo volver a su lugar de origen.

Inicialmente estas necesidades se satisfacieron en cada época de acuerdo a los medios científicos y tecnologías disponibles. Se pasó en un principio de simples chozas y dibujos ó esquemas elementales sobre tablillas de barro, hasta los sofisticados sistemas actuales que aúnan técnicas nuevas, como la robótica, los

This paper deals with of a the use of Geographical Information Systems (GIS) for domotic control. The foccus is puton the communication interface between the building control system (BCS) integrated in a GIS. For get this aim, the GIS of the Montegancedo Campus where is located the Facultad de infomatica of UPM and the creation of an interface is needed. The implemented interface in Microsoft C\# language allows the control, monotorizing and management of the sensors data installated in the Campus.

Keywords: Geographic Infomation Systems (GIS), Domotic, Buildings control, Sensors.

sistemas domóticos de control de edificios, el posicionamiento por satélite, la teledetección, Internet, etc.

En la actualidad, se está asistiendo a la explosión de lo que se denomina edificios inteligentes o digitales cuya definición ha evolucionado en los últimos años. Se define como edificio inteligente un ambiente estructurado, equipado de varios sistemas de sensores y de control, destinado a actividades de monitorización y control (dentro o fuera del edificio), empleando infraestructuras de red heterogéneas que hacen posible la comunicación entre los sistemas entre si y con el ser humano (1). Es un centro de comunicaciones que permite estar conectado con el exterior y donde interactuan

\footnotetext{
(*)Facultad de Informática (U.P.M.), Boadilla del Monte, Madrid (España)

${ }^{(* *)}$ CISDEM: Centro de Inv. en Seguridad y Durabilidad Estructural y de Materiales (CSIC-UPM)

Persona de contacto/Corresponding author: malvarez@fi.upm.es (M. Álvarez)
} 
entre sí multiples dispositivos tanto situados en el interior como en el exterior y en cualquier parte del mundo. Presentan unas características principales que los determinan (2): el control remoto desde el interior del edificio, el acceso a servicios externos, la programabilidad y el control remoto desde el exterior.

Las dificultades que se presentan para la instalación de un sistema de este tipo van desde, el coste final, el ser un área multidisciplinaria en la cual deben trabajar de forma complementaria expertos de diferentes campos, la existencia de distintos protocolos y la incompatibilidad en el intercambio entre formatos de los datos que maneja. La resolución de la mayoría de estos problemas, integración de formatos entre programas, sensores, etc., puede ser resuelta con la utilización de un SIG. Tradicionalmente los SIG, que permiten abstraer la realidad como un objeto y modelizarla usando estructuras simples, se han utilizado para: definir qué hay en una determinada localización, encontrar terrenos apropiados para un determinado fin, establecer las diferencias temporales de un lugar, realizar distribuciones estadísticas que permitan identificar cualquier relación espacial con determinados incidentes, por ejemplo, una distribución de los delitos por barrios de una ciudad y modelizaciones $(3,4)$.

En la actualidad un SIG se puede definir como un sistema de seis componentes: Hardware, corre sobre diversos tipos de arquitecturas, dispositivos moviles, servidores de web de cartografia, equipos de mesa, etc.; software; datos; procedimientos para el análisis, representación y gestión de una gran variedad de tipos y formatos de datos; organización que agrupa el equipo multidisciplinar que debe manejarlos e Internet. Parece ser una tecnología que podría resolver los problemas arriba mencionados que conlleva un sistema domótico.

La integración en los SIG de datos procedentes de sensores es bien conocida y usada desde hace años. Los SIG manejan información de sensores remotos de teledetección (5), de radares, de datos procedentes de GPS, de sensores que miden descargas eléctricas atmosféricas

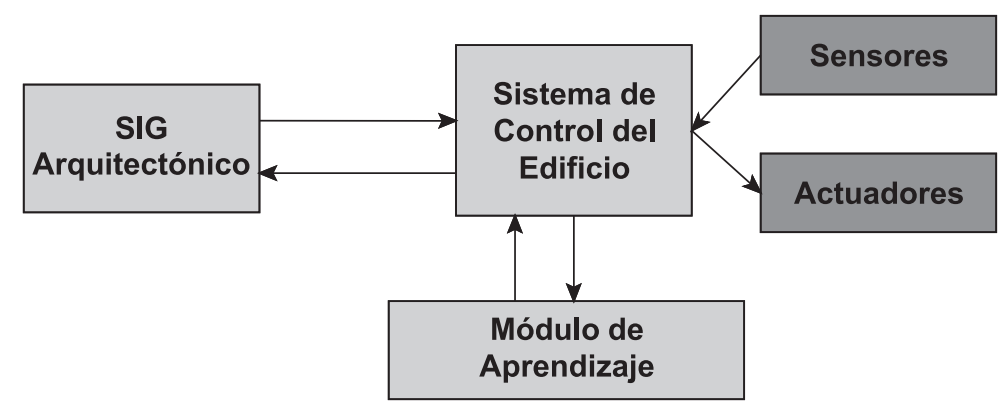

[web1], de sensores terrestres para enviar alarmas en proyectos de tunelación, etc.

La base de datos del SIG contiene toda la información necesaria para describir las características relevantes del edificio y sus instalaciones. Permite una gestión integrada de la información, la inserción y el borrado de datos, de nuevos conjuntos que los contengan y de tablas. También es posible añadir tablas de relaciones a la base de datos (como en una base de datos relacional común) que permitan efectuar operaciones más complejas sobre los datos y la obtención de nuevos.

El sistema que se muestra en la Figura 1 representa una idea del posible esquema general de un sistema domotico a desarrollar. El centro del sistema es el SIG Arquitectónico al que se llama Modelo del Edificio. En él están almacenadas la disposición todas las plantas del Campus y los distintos edificios con la descripción completa de las instalaciones a controlar.

Otro componente del sistema es el Sistema de Control de un Edificio (SCE) usado para realizar el control y obtener nueva información en tiempo real actuando sobre las instalaciones. El sistema de control avanzado de un edificio debe ser flexible y abierto, debido a que un edificio es un sistema con una evolución continua y, en algunos aspectos, todavía poco conocida. El sistema de control debe ser capaz de evolucionar, adaptándose a los cambios que se producen en el edificio, retroalimentandose con los datos de su propio funcionamiento y utilizarlos, para mejorar su rendimiento.

Es necesaria la existencia de un interfaz, programa informático que permita al sistema comunicarse con el usuario (IS) o con otras aplicaciones, integrado en el SIG, que posibilite la comunicación con el sistema de control del edificio. Asimismo, la facilidad y sencillez de desarrollo de un servidor web que proporcione datos y servicios SIG, puede permitir la monitorización remota para el acceso a los servicios del edificio y un servicio de alarmas técnicas, desde otros lugares convenientes.

El trabajo que se expone consiste, en primer lugar, en desarrollar una aplicación SIG del Campus de Montegancedo y de la Facultad de Informática de la UPM. Este SIG contiene todos los datos del campus y sus edificios, las plantas, cubierta, fecha de construcción, número de locales en cada planta, superficies por local, situación de las instalaciones, calefacción, zonas de iluminación, información de sensores de los locales, dispositivos de control de los mismos, etc.

En segundo lugar se presenta el desarrollo de una versión DEMO de un interfaz integrado 
en el SIG que implementa algunas funciones domóticas.

\section{METODOLOGÍA}

La metodología de desarrollo de este proyecto comprende dos etapas. Cada una de ellas se descompone, a su vez, en una serie de fases.

\section{Diseño del SIG.}

2. Integración del SIG con un Sistema de Control del Edificio (SCE).

\subsection{Diseño del SIG}

La necesidad del personal de la Facultad de Informática de la UPM de poseer una herramienta para gestionar sus propios edificios y parte del Campus de Montegancedo donde está situada, condujo a las autoras de este trabajo a desarrollar un SIG del mismo. Este SIG permitirá gestionar cualquier dato de información arquitectónica, ya sea gráfico o alfanumérico, de manera rápida, cómoda, eficaz y sencilla.

En total, las dimensiones de la zona a incluir en el SIG son, aproximadamente, un kilómetro de largo por unos 700 metros de ancho, en su punto máximo. En toda esa extensión, existen grandes zonas sometidas a protección medioambiental, por presentarse restos de bosque mediterráneo con la presencia de arbolado compuesto de encinas y pinos, junto con el matorral característico del que forma parte, con una densidad considerable, el matorral de jara pringosa.

Está dividido, territorialmente, entre los términos municipales de Pozuelo de Alarcón y Boadilla del Monte, con la propiedad dividida entre la Facultad de Informática, propietaria de una zona con 3 edificios de aulas, despachos, administración y zona de aparcamiento propia, y la UPM, propietaria de la mayor parte del Campus. Esta doble pertenencia dificulta la obtención de datos geográficos de todo el
Campus, pues es necesario dirigirse a dos organismos responsables distintos. Además, como así ocurrió, la diferencia en los formatos de los datos obtenidos impide su tratamiento conjunto. En un principio se realizó un SIG 2D del Campus, de dimensión en memoria cercana a $8 \mathrm{Mb}$, y en una segunda fase se procedió a desarrollar un SIG 3D. El diseño del SIG está estructurado en varios módulos (6), como se indica en la Figura 2:

- Módulo Esquema.

- Módulo General.

- Módulo de Explotación.

\subsubsection{Módulo Esquema}

En este módulo se realiza el análisis y el proceso de unificación de formatos de los distintos tipos de datos. También se incluye el proceso de digitalización de la información que no está en formato digital. Consta a su vez de dos fases:

- Estudio del Software SIG a utilizar.

- Selección de los datos.

\section{Estudio del Software SIG a utilizar}

Existen numerosos programas y paquetes de aplicaciones de SIG comerciales y de libre distribución. Para este trabajo y como consecuencia de las mejores capacidades que ofrece se escogió ArcGIS, una arquitectura de productos software cuya unión crea un SIG completo desarrollado por la empresa ESRI [web 2] que en la actualidad es el líder del mercado, tiene muchas funcionalidades, proporciona un mayor soporte al usuario y permite una gran interoperabilidad de formatos. El objetivo es poder obtener ficheros vectoriales definidos, bien dibujando directamente o sobre las imagenes ráster, rectificadas o no.

Dado que ArcGIS es compatible con los formatos raster más comunes, se utilizó para ellos el formato shape (vectorial). En este formato se introdujeron las imágenes remotas clasificadas temáticamente.
2. Diagrama de flujo de los módulos

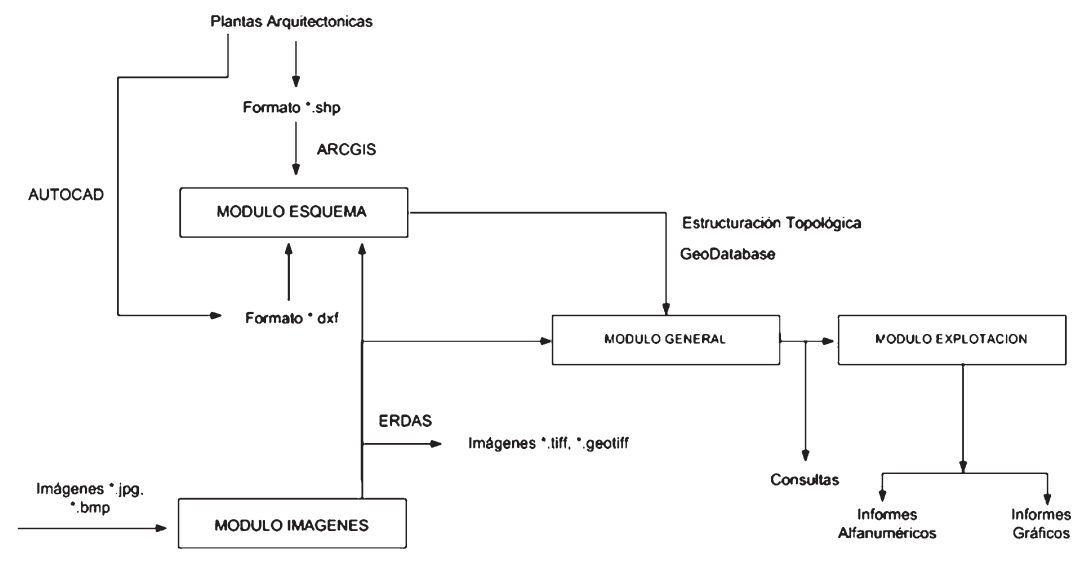




\section{Selección de los datos}

Como el fin del desarrollo del SIG era su posibilidad de uso por la FI (UPM) en diferentes tipos de aplicaciones, de gestión, arquitectónicas, medioambientales, etc., la información que contiene es muy variada, tanto por su procedencia, como por el medio de adquisición.

Se ha contado con los siguientes datos:

Datos del Campus en formato de AutoDesk, [web 3] procedentes del levantamiento realizado por el servicio de infraestructuras del Rectorado de la UPM.

Datos de los edificios en formato .dxf de AutoCAD, proporcionados por la dirección de la Facultad de Informática.

Imágenes, necesarias a la hora de ubicar visualmente los datos y entender el contexto en el que son presentados. Se han utilizado varios tipos de imágenes:

Fotografías aéreas, se utilizó una fotografía aérea en formato GeoTIFF (formato de imágenes georreferenciadas) proporcionada por el Centro Geográfico del Ejército (CGET).

Esta foto aérea no estaba actualizada y tras una verificación de los datos en una campaña de campo, se han podido incluir las plantas de los nuevos edificios que se están construyendo en el Campus, Figura 3:

- Edificio CESVIMA: Vivero de empresas.

- Edificio Centro de Investigación en Domótica Integral (CDINI).

- Instituto de Biotecnología y Genómica de plantas.
- Laboratorio de plantas.

- Edificio USOC.

Fotografías de los edificios, imágenes de los edificios que se usaron para modelar los edificios en 3D. Se empleó un mosaico de fotografías tanto del interior como del exterior de los edificios y su fachadas.

Imágenes remotas, en este trabajo se ha utilizado una subescena $\left(1.200 \times 1.200 \mathrm{~m}^{2}\right)$ de una imagen del satélite de teledetección LANDSAT $E M T+$, clasificada mediante un método supervisado incluido en el programa de tratamiento de imágenes ErMapper, en 6 clases temáticas de ocupación del suelo, Figura 4.

Modelo Digital del Terreno (MDT), se dispuso de un modelo digital del terreno con una precisión de 25 metros. La información no estaba correctamente georreferenciada y fue necesario retocarla mediante scripts de tratamiento espacial. Se consideró conveniente el uso de técnicas de almacenamiento como Pirámides, debido a la elevada carga computacional, que permitieron obtener un mejor resultado en cuanto a los tiempos de respuesta.

\subsubsection{Módulo Genera}

Este módulo se encarga de organizar, gestionar y mantener toda la información, vinculando el resto de módulos. Como Sistema Gestor de la Base de Datos (SGBD) se ha utilizado Personal Microsoft Acceess. El SGBD Espacial es $A R C S D E$ de Esri, que constituye una capa por encima del relacional. Tiene como objetivo hacer de interfaz a las aplicaciones que deseen gestionar la información gráfica que hay almacenada en la base de datos. Puede almacenar todo tipo de información: vectorial, raster, pro-

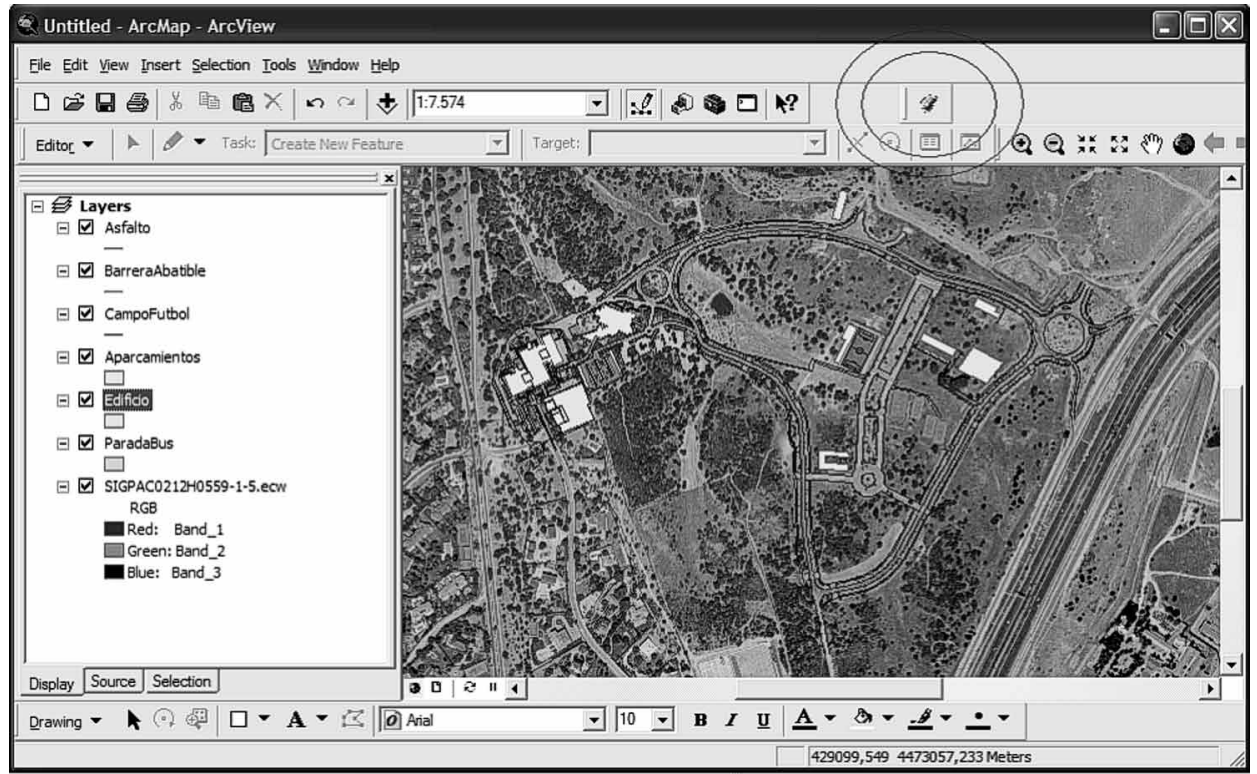


cedente de sensores de medidas de campo y topográficos, datos CAD, 2D y 3D, etc.

El SIG se estableció con una estructura jerárquica de los ficheros del proyecto, que permite la referenciación entre distintos elementos del proyecto. Esta estructura se compone de un nodo raíz, que contiene dos geodatabases, una denominada "Alumno", otra denominada "Mantenimiento" los archivos .mxd para cada usuario del SIG y una carpeta, denominada "img", que contiene las imágenes asociadas a ciertos elementos de las capas. La razon de crear dos geodatabases distintas fué permitir la distribución y uso de dichos datos orientados hacia el uso que se les va a dar.

En la estructura de directorios del ordenador, cada geodatabase, con todo su contenido, aparece como un archivo único, "Alumno.mdb" y "Mantenimiento.mdb", que puede ser visualizado y editado con herramientas como Microsoft Access o bien con la aplicación ArcCatalog de ArcGIS.

Dependiendo de la utilidad que se vaya a dar a la información, se seleccionaron las capas temáticas a incluir en las bases de datos, y sus tablas alfanuméricas relacionadas. De este modo, la base de datos de "Mantenimiento" además de las capas de la de "Alumno" contiene otras necesarias para el mantenimiento del Campus y una tabla de atributos para complementar al raster de clasificación temática incluido en el SIG. La base de datos "Alumno" esta compuesta de las capas necesarias para cubrir las necesidades de la FIM respecto a los alumnos de la Facultad

Tras la integración de todos los datos, se ha obtenido un conjunto de 70 capas formadas por datos vectoriales y dos capas raster, "radiometría Landsat" y "radiometría aérea". Cada plano, se organizó por capas y cada capa tiene asignadas las propiedades habituales en un sistema de información como el color, el grosor, y unidades de medida. Esta simbología se puede cambiar fácilmente.

La clasificación en capas permite desarrollar el concepto de colecciones de datos temáticos datasets que pueden representar, bien información recogida o interpretada, o bien datos resultantes de operaciones de análisis y modelización. Pueden ser creados y gestionados como información genérica que puede ser compartida entre varios usuarios a través de la web.

Es usual que los SIG usen varios datasets con representaciones procedentes de varias organizaciones. Además, es interesante señalar que en un mismo dataset es posible almacenar capas que utilicen diferentes tipos de formatos. A

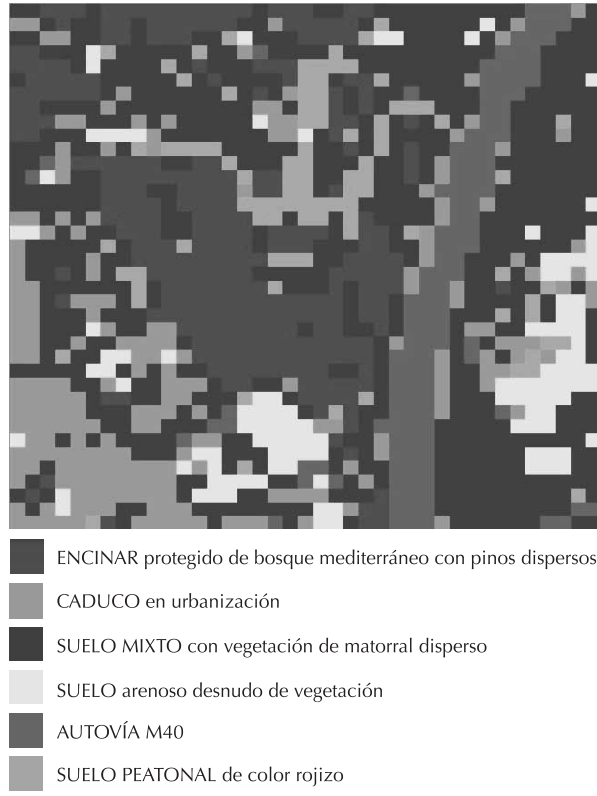

4. Imagen LANDSAT EMT+, clasificada.doc. modo de ejemplo, la base de datos denominada "Alumno" contiene dos dataset, el conjunto llamado Edificio 1_6 donde se encuentran los planos de los edificios de la Facultad de Informática estructurados en plantas. Otras características del Campus que pueden interesar a los alumnos estan agrupadas en el conjunto Servicios.

La generación de los datos del SIG presentó mas problemas de los inicialmente esperados. La exportación de datos desde AutoDesk no fue perfecta debido a la imposibilidad de exportar ciertas capas, y a que alguna de las capas exportadas presentaban errores. Debido a ello en vez de exportar desde AutoDesk, se acabó recurriendo a la importación desde el propio ArcGIS de los datos CAD.

Tambien fue necesario editar información para corregir los errores de los archivos CAD originales, para añadir elementos nuevos detectados en campañas de campo, y para añadir las capas que fue necesario crear a mano sobre la base del archivo.$d w g$, tarea que resultó extremadamente laboriosa, ya que no sólo hubo que llevar a cabo un proceso de edición, sino también de corrección. Una vez generados los datos se procedió a exportarlos para la realización de la aplicación 3D mediante el software Sketch de Google y a partir de ellas se pudo dotar al escenario de un mayor realismo, véase la Figura 5, pág. siguiente.

\subsubsection{Definición del Módulo de Explotación}

El usuario podrá elegir entre todas las funciones posibles de un SIG.

Entre otras se pueden realizar las siguientes funciones: 

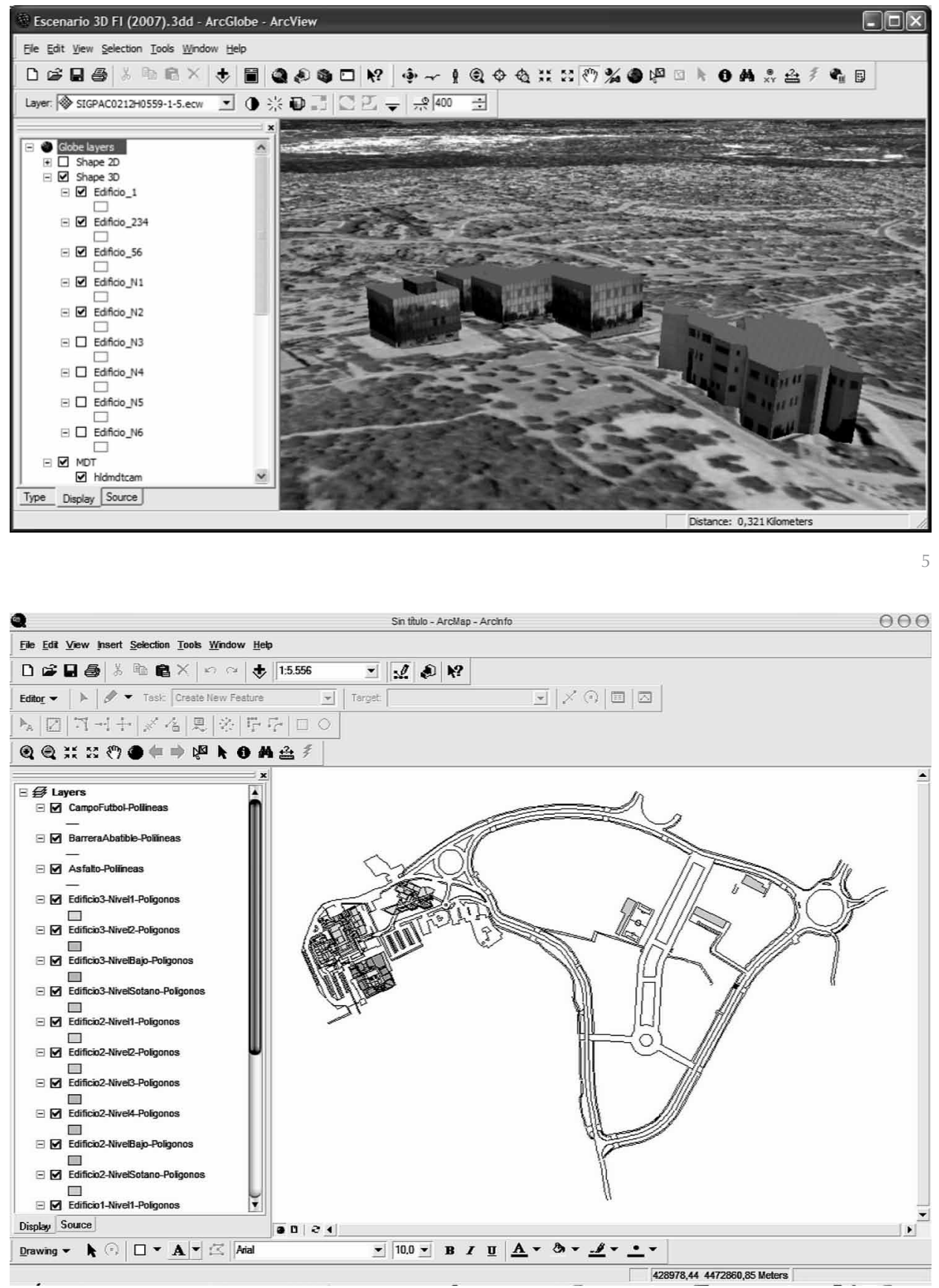

- Realizar consultas con la información alfanumérica utilizando el asistente que incorpora el propio programa o mediante lenguaje $S Q L$ de consulta a la base de datos.

- Visualizar las consultas en el entorno gráfico. En la Figura 6, se muestra una visualización de la pantalla principal de la aplicación. En la ventana de la izquierda se muestran todas las capas y en la de la derecha se visualiza la capa seleccionada, en este caso, la capa edificios.
El programa permite realizar todo tipo de zoom, ventana, anterior, extensión. Todas las visualizaciones de los planos podrán imprimirse en cualquier momento.

\subsection{Integración del SIG con un Sistema de Control del Edificio (SCE).}

Existen multitud de sensores en el mercado de la domótica, que situados en los edificios pueden interactuar con el Sistema de Control del Edificio.

Las funciones más frecuentes a controlar a través de sensores se pueden clasificar en:

- Automatización y Control, incluye el control (abrir / cerrar, on / off y regulación) de la iluminación, climatización, persianas y toldos, puertas y ventanas, cerraduras, riego, electrodomésticos, suministro de agua, gas, etc. 
- Seguridad, incluye alarmas de intrusión, alarmas personales y alarmas técnicas (incendio, humo, agua, gas, fallo de suministro eléctrico).

- Telecomunicaciones, incluye transmisión de voz y datos con redes locales (LAN) para compartir acceso de alta velocidad a Internet, recursos y el intercambio entre todos los equipos. Además permite disfrutar de nuevos servicios como Telefonía sobre IP y Televisión Digital.

- Audio y vídeo, incluye la distribución de imágenes de video capturadas con cámaras dentro y fuera de la casa a toda la casa y a través de Internet. Otra parte de audio / vídeo trata del entretenimiento como el "multi-room" y el "Cine En Casa".

\subsubsection{Prototipo del interfaz}

Dentro de la implementación se ha realizado una versión DEMO que cumple las necesidades de versión prototipo de la interfaz. Con esta versión DEMO se pretende realizar un programa capaz de controlar algunas funciones en instalaciones existentes en el Campus y los edificios, y que tenga un manejo sencillo de la información que se dispone.

Como herramienta para implementar la aplicación se ha usado el lenguaje de programación de alto nivel C\# incluido entre los lenguajes del entorno Visual Studio. NET proporcionado por Microsoft. Se ha usado este entorno debido a que resulta más intuitivo y completo que otros entornos enbedded. Debido al uso de C\# y a los entornos .NET, esta DEMO puede incluirse en cualquier poyecto SIG para dispositivos móviles que use dicho entorno y su traslado a C++ resulta sencillo por la similitud de sintaxis existente entre estos lenuajes de alto nivel.

\subsubsection{Funcionamiento de la aplicación}

Al iniciar la aplicación, Figura 7, pág. siguiente, se muestra un plano de todos los elementos del SIG. Sobre él, el usuario puede elegir el edificio, planta o instalación que quiere controlar. El programa permite hacer uso de la herramienta zoom para una mejor visualización.

El propio interfaz dirige al usuario para que realice las tareas separando, según la función a controlar, en dos o tres pasos el uso del mismo. En el paso 1 se carga el indicador de la opción a controlar. Para ello se pulsa sobre el botón aceptar. Una vez pulsado emerge una segunda ventana cuyo objetivo es mostrar las condiciones actuales de la función que se está controlando.

Aceptando sobre el botón correspondiente aparece una tercera ventana cuyo objetivo es permitir cargar las condiciones deseadas en ese momento de la función a controlar.

Entre todas las funciones posibles de control, el programa permite realizar las siguientes:

- Activar el sistema de riego

- Detección de personas

- Control de climatización

- Control de la luz natural

\subsubsection{Activar el sistema de riego}

Esta función permite activar el sistema de riego del campus a una hora determinada. En este caso, a modo de ejemplo, se muestra el control del riego del campo de fútbol del campus.

El usuario seleccionará en el SIG las instalaciones deportivas. El interfaz cargará un menú con todas las posibles acciones que puede realizar el usuario, visualizando un 3D de las instalaciones deportivas, Figura 8, pág. siguiente.

El usuario seleccionará el "Indicador de la Opción de riego" pudiendo modificar la fecha y la hora para que se inicie, Figura 9, pág. siguiente. Esto activará los sensores que controlarán el riego del campo de fútbol a la hora propuesta.

\subsubsection{Detección de personas}

Esta función permite saber cuál es el número de personas que se encuentra en un determinado local en un determinado momento.

En este caso se trata de detectar el número de personas que se encuentran en el aula 3101.

El usuario tiene dos opciones de selección del aula: visualizando en el SIG la planta donde está el aula que desea controlar, o introduciendo los datos del bloque donde está situada, Figura 10, pág. 23.

A continuación se selecciona el "Indicador de Presencia", Figura 11, pág. 23 y el sistema podrá leer la información que le devuelva el sensor situado en el aula y mostrar por pantalla la información al usuario del número de personas presentes, Figura 12, pág. 23.

\subsubsection{Control de climatización}

Esta función permite al usuario controlar la temperatura existente en un momento determinado en un local para poder aumentarla o disminuirla. En este caso se trata de controlar la temperatura del aula 3101. El usuario señalará en el interfaz la planta y el número de aula y, 
7. Pantalla de inicio de la aplicación.

8. Plano 3D de las instalaciones deportivas.

9. Indicador de Opción de Riego.

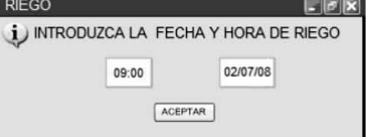

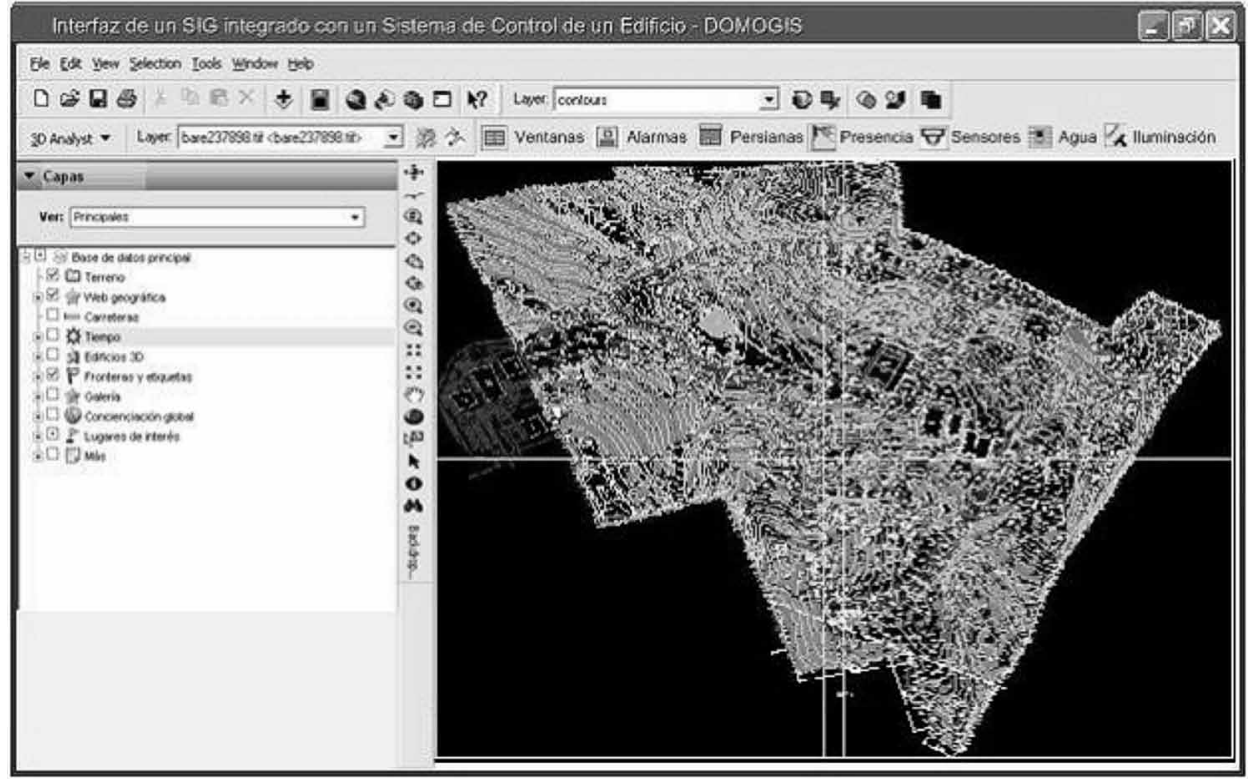

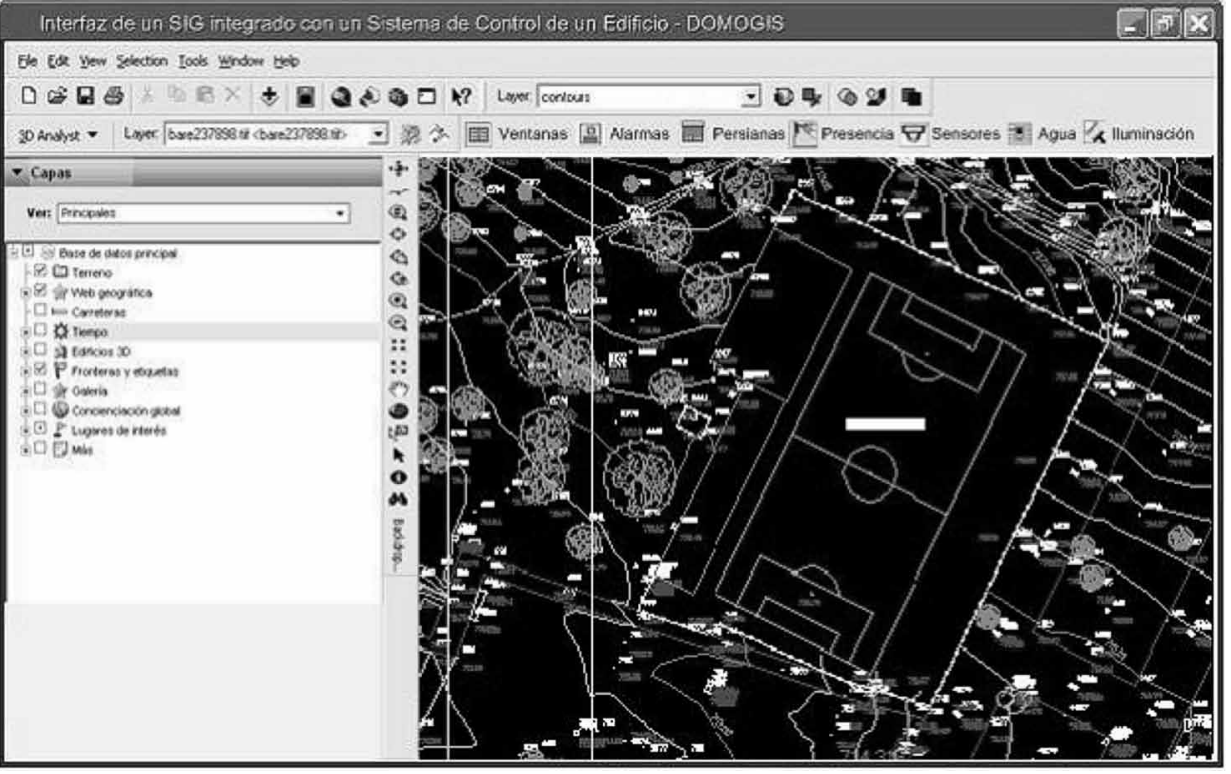

a continuación, seleccionará el "Indicador de Temperatura", Figura 13, pág. 24. El sistema leerá la información que le devuelva el sensor situado en el aula y muestra por pantalla la información de la temperatura del aula, Figura 14, pág. 24. Si el usuario desea elevar o disminuir la temperatura del aula 3101, tan sólo tendrá que acceder al menú de Temperatura y mediante el uso de los botones, indicar cuál es la nueva temperatura deseada. El sistema lee la información que le devuelva el sensor situado en el aula y muestra por pantalla la información de la temperatura, Figura 15, pág. 24.

\subsubsection{Control de la luz natural de un aula} utilizando el indicador Persianas

En este caso, el usuario seleccionará en el SIG la ventana del local cuya iluminación desea contro- lar. A continuación accederá al menú "Indicador de Persianas". Seleccionará el nivel de iluminación que desea, modificando el nivel de iluminacion natural del local, tal y como puede observarse en

\section{CONCLUSIONES}

1. La creación de un SIG es especialmente útil para controlar, gestionar, manejar, actualizar y analizar toda la información geográfica y alfanumérica de un organismo como la UPM, debido a la posibilidad y facilidad para actualizar los datos, generar nueva información, calcular recorridos, apreciar zonas donde construir, etc.

2. En lo referente a la realización del SIG, aunque conceptualmente no presenta la Figura 16 de la página 24. 


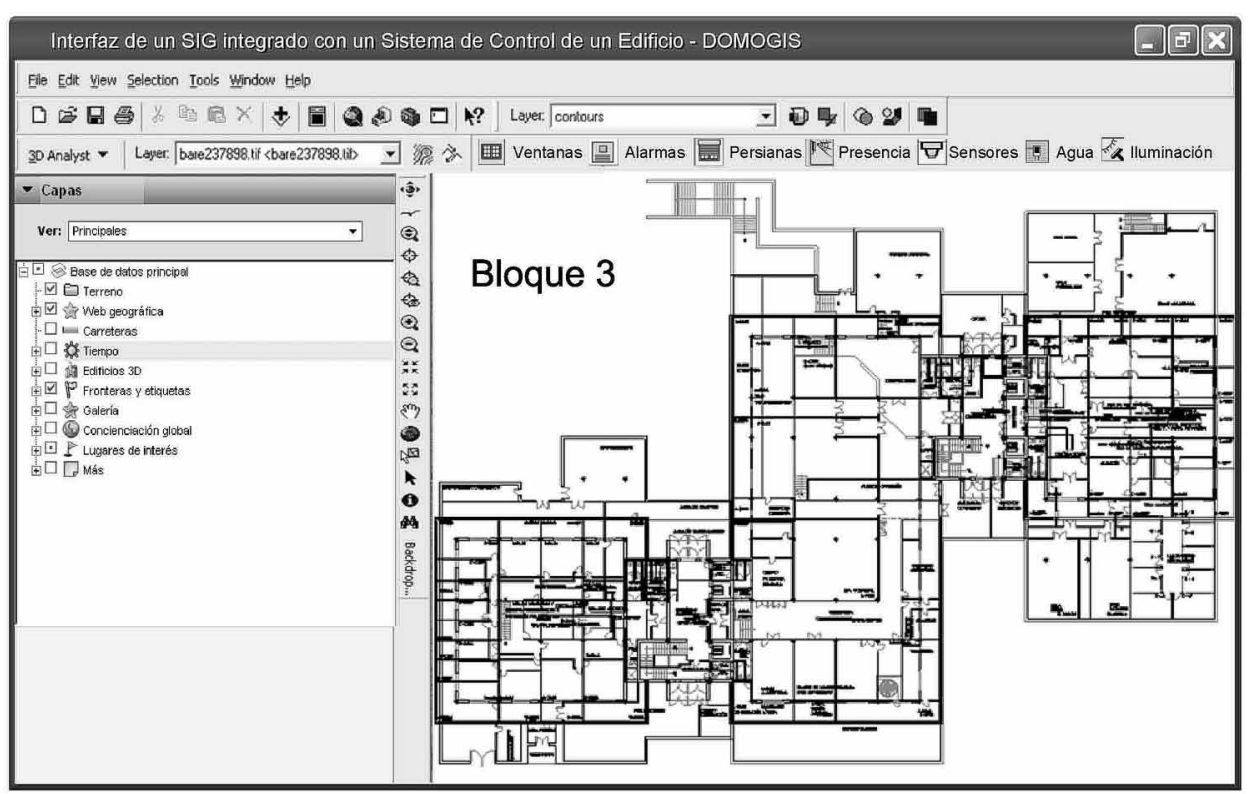

10

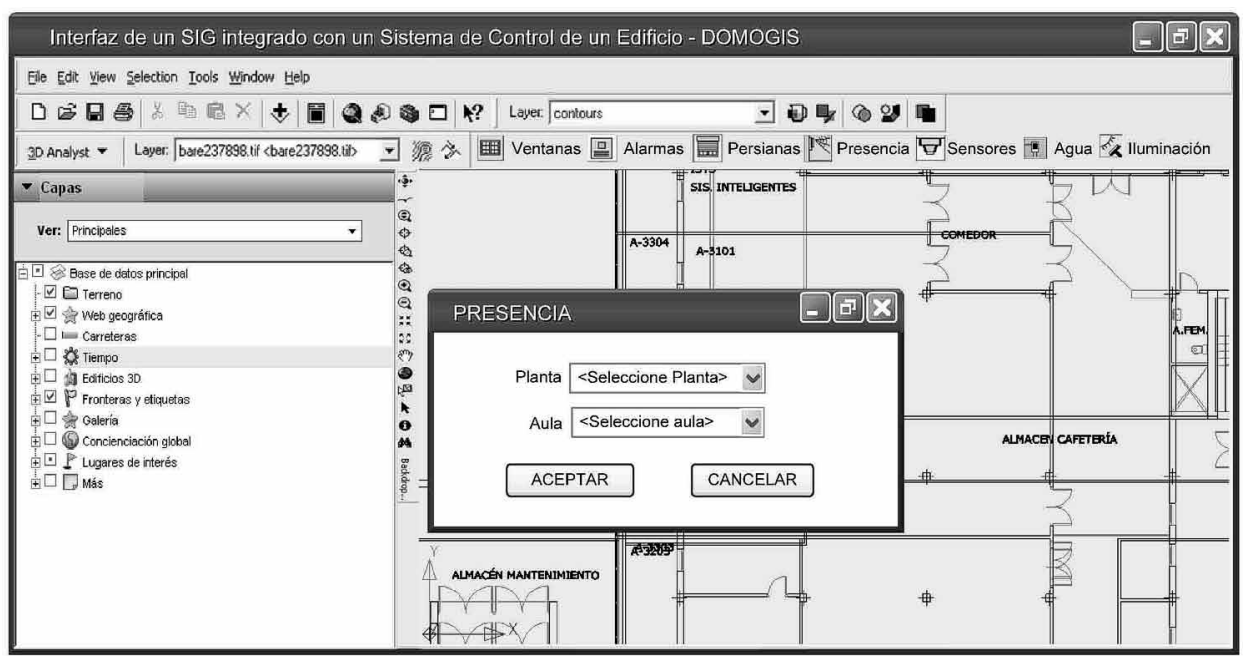

muchas dificultades, se basa en principio en conseguir un SIG de calidad a partir de los datos CAD de entrada, hay que indicar que presentó más dificultad de lo esperado.

3. Se aceptó el tipo de capas de elementos de origen, verificándolas, adaptándolas y ampliándolas en algunos casos. Se han añadido capas nuevas, tanto raster como vector.

4. Se presenta, a modo de ejemplo, un prototipo de una herramienta de ayuda a la creación de sistemas de control basada en la utilización de un SIG. Permite comparar los parámetros utilizados para la adaptación del sistema a los SCE de diferentes edificios y la reutilización de los diseños aplicados en el control de las instalaciones de edificios previos.

5. Sobre el SIG, se desarrolló una interfaz inicial, una herramienta fácil de usar y flexible a cambios, que permite la conexión al Sistema de Control del Edificio.

6. La realización de la herramienta, aunque conceptualmente es más compleja que el SIG, no presentó ninguna dificultad en su funcionalidad básica. En un futuro convendría mejorar tanto la funcionalidad ofrecida, como el propio interfaz para adaptarlo a un cliente web, lo que supondría una simplificación de tareas al permitir acceder por control remoto.

7. Se pueden usar sobre otros tipos de arquitecturas SIG como dispositivos electrónicos de bolsillo, PDAs, etc.

\section{AGRADECIMIENTOS}

Este articulo no habria sido posible sin el esfuerzo y la participacion de los alumnos de la FIM (UPM): Ruben Cogollor, David Corpas y Victor Uriel.
10. Plano de situación del aula que se desea controlar.

11. Indicador de Presencia

12. Resultado del Indicador de Presencia. (i) El número de personas detectado en el Aula 3101 es de $\stackrel{30}{{ }_{A C E P T A R}}$ 
13. Indicador de Temperatura.

14. Resultado del Indicador de Temperatura

15. Control de cambio de Temperatura.

16. Indicador de control de iluminación de Persianas.
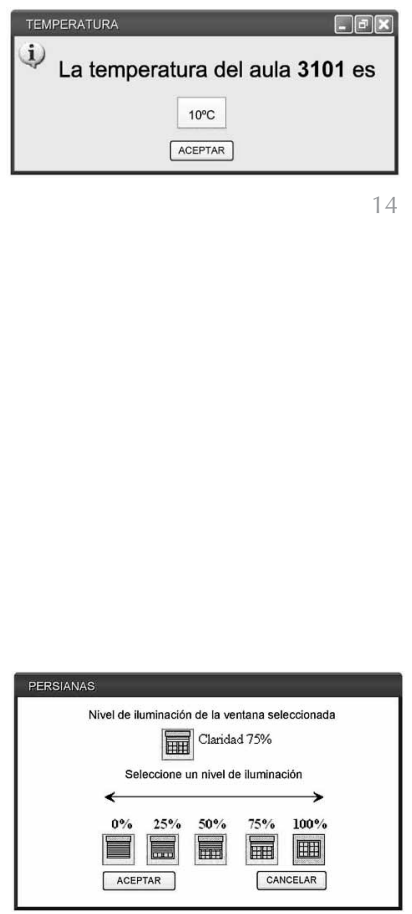

16

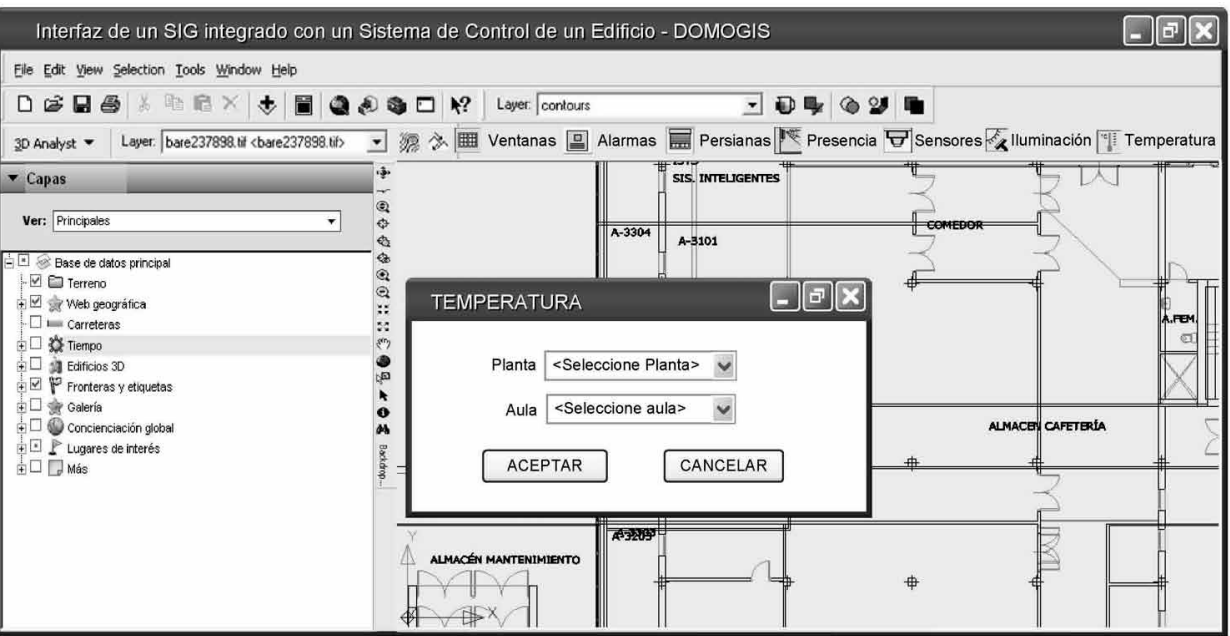

Interfaz de un SIG integrado con un Sistema de Control de un Edificio - DOMOGIS

Ele Edit: Wiew Selection Iools window Help

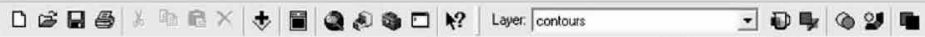

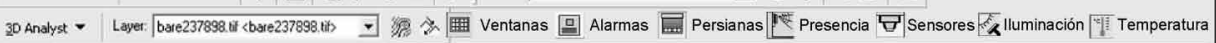

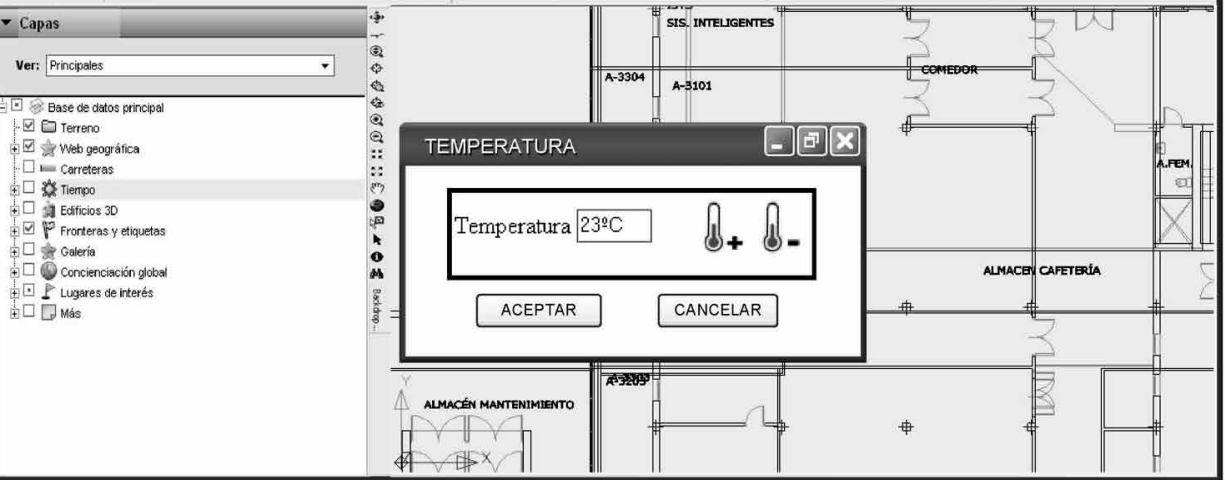

15

\section{BIBLIOGRAFÍA}

(1) Triviño, G.: "Una propuesta para la arquitectura informática del sistema de control de un edificio", Informes de la Construcción, CSIC, Vol. 56, nº 494, 2004.

(2) Florez, M. A.: "Hacia una definición de la domótica", Informes de la Construcción, CSIC, Vol. 56, n 494, 2004.

(3) Arquero, A.; Álvarez, M.; Martínez, E.: Decision Management Making by AHP (Analytical Hierarchy Process) trought GIS data, IEEE Latin America Transactions Rev., Vol. 7, n 1. 2009.

(4) Álvarez, M.; Arquero, A.; Martínez, E.: "Empleo del AHP incorporado en SIG para definir el emplazamiento optimo de equipamientos universitarios. Aplicación a una biblioteca", XII Congreso Nacional de Tecnologías de la Información Geográfica, Granada, 2006.

(5) Arquero, A.; Gonzalo, C.; Martínez, E.: "Teledetección. Una aproximación desde la superficie al satélite", Fundación General de la UPM, 2003.

(6) Núñez, A.; Buill, F.; Lantada, N.; Calaf, F.: Sistema de Información Arquitectónica (S.I.A.), IX Conferencia Iberoamericana de Sistemas de Información Geográfica. Cáceres, 2003.

(7) Triviño, G.: "El edificio como un sistema de gestión de la información", Informes de la Construcción, CSIC, Vol. 55, no 488, 2003.

Web:

[Web 1] ZEUS: http://gis.esri.com/library/userconf/latinproc99/ponencias/ponencia43.html

[Web 2] Esri es una de las compañías más importantes de desarrollo SIG.

Disponible en: http://www.esri.com/

[Web 3] El producto AutoCAD es de la empresa Autodesk.

Disponible en: http://www.autodesk.es/adsk/servlet/index?sitelD=455755\&id=10480648 